The Journal of $\mathbf{N}_{\text {onlinear }} \mathbf{S}$ cience and Applications http://www.tjnsa.com

\title{
SECOND ORDER CONVERSE DUALITY FOR NONLINEAR PROGRAMMING
}

\author{
I. AHMAD ${ }^{1, \dagger}$ AND RAVI P. AGARWAL ${ }^{1, \ddagger *}$
}

Abstract. Chandra and Abha [European J. Oper. Res. 122 (2000), 161-165] considered a nonlinear programming problem over cone constraints and presented the correct forms of its four types of duals formulated by Nanda and Das [European J. Oper. Res. 88 (1996) 572-577]. Yang et al. [Indian J. Pure Appl. Math. 35 (2004), 699-708] considered the same problem and discussed weak and strong duality for its four types of second order duals under the assumptions of generalized second order F-convexity. In this paper, we are intended to prove converse duality theorems for second order duals of Yang et al.

\section{Introduction}

Nanda and Das [3] considered the following nonlinear programming problem with cone constraints:

Problem (P)

Date: Received: 28 Oct. 2010.

* Corresponding author

(c) 2010 N.A.G.

2000 Mathematics Subject Classification. 90C30; 90C46.

Key words and phrases. Nonlinear programming; second order duality; converse duality; cone constraints.

${ }^{\dagger}$ Permanent address: Department of Mathematics, Aligarh Muslim University, Aligarh- 202 002, India.

${ }^{\ddagger}$ Permanent address: Department of Mathematical Sciences, Florida Institute of Technology, Melbourne 32901, USA. 
Minimize $f(x)$

subject to $g(x) \in C_{2}^{*}$

$x \in C_{1}$,

and formulated four types of dual models associated to (P). Chandra and Abha [2] removed certain omissions and inconsistencies in the dual formulations of Nanda and Das [3]; they presented following correct forms of these duals as follows:

Problem (D1)

Maximize $f(u)+y^{t} g(u)-u^{t} \nabla\left(f+y^{t} g\right)(u)$

subject to $-\nabla\left(f+y^{t} g\right)(u) \in C_{1}^{*}$,

$y \in C_{2}$.

Problem (D2)

Maximize $f(u)$

subject to $-\nabla\left(f+y^{t} g\right)(u) \in C_{1}^{*}$

$y^{t} g(u)-u^{t} \nabla\left(f+y^{t} g\right)(u) \geq 0$,

$y \in C_{2}$.

Problem (D3)

Maximize $f(u)-u^{t} \nabla\left(f+y^{t} g\right)(u)$

subject to $-\nabla\left(f+y^{t} g\right)(u) \in C_{1}^{*}$,

$y^{t} g(u) \geq 0$,

$y \in C_{2}$.

Problem (D4)

Maximize $f(u)+y^{t} g(u)$

subject to $-\nabla\left(f+y^{t} g\right)(u) \in C_{1}^{*}$,

$u^{t} \nabla\left(f+y^{t} g\right)(u) \leq 0$ 
$y \in C_{2}$.

In continuation with the weak and strong duality theorems proved by Chandra and Abha [2], Yang et al. [5] established the converse duality theorems between (P) and (D1), (D2), (D3) and (D4), respectively. In [4], Yang et al. constructed following four types of second order duals associated to $(\mathrm{P})$, which are obviously the second order extension of above duals (i.e., (D1), (D2), (D3) and (D4)). In order to relate (P) with (ND1), (ND2), (ND3) and (ND4), they proved weak and strong duality theorems under generalized second order F-convexity assumptions. But, the converse duality was not dealt in their treatment.

Problem (ND1)

Maximize $f(u)+y^{t} g(u)-\frac{1}{2} p^{t} \nabla^{2}\left(f(u)+y^{t} g(u)\right) p$

$$
-u^{t}\left[\nabla\left(f(u)+y^{t} g(u)\right)+\nabla^{2}\left(f(u)+y^{t} g(u)\right) p\right]
$$

subject to $-\left[\nabla\left(f(u)+y^{t} g(u)\right)+\nabla^{2}\left(f(u)+y^{t} g(u)\right) p\right] \in C_{1}^{*}$,

$y \in C_{2}$.

Problem (ND2)

Maximize $f(u)-\frac{1}{2} p^{t} \nabla^{2} f(u) p$

subject to $-\left[\nabla\left(f(u)+y^{t} g(u)\right)+\nabla^{2}\left(f(u)+y^{t} g(u)\right) p\right] \in C_{1}^{*}$,

$$
\begin{aligned}
& y^{t} g(u)-\frac{1}{2} p^{t} \nabla^{2} y^{t} g(u) p-u^{t}\left[\nabla\left(f(u)+y^{t} g(u)\right)+\nabla^{2}\left(f(u)+y^{t} g(u)\right) p\right] \geq 0, \\
& y \in C_{2} .
\end{aligned}
$$

\section{Problem (ND3)}

Maximize $f(u)-\frac{1}{2} p^{t} \nabla^{2} f(u) p-u^{t}\left[\nabla\left(f(u)+y^{t} g(u)\right)+\nabla^{2}\left(f(u)+y^{t} g(u)\right) p\right]$

subject to $-\left[\nabla\left(f(u)+y^{t} g(u)\right)+\nabla^{2}\left(f(u)+y^{t} g(u)\right) p\right] \in C_{1}^{*}$,

$$
\begin{aligned}
& y^{t} g(u)-\frac{1}{2} p^{t} \nabla^{2} y^{t} g(u) p \geq 0, \\
& y \in C_{2} .
\end{aligned}
$$

Problem (ND4)

Maximize $f(u)+y^{t} g(u)-\frac{1}{2} p^{t} \nabla^{2}\left(f(u)+y^{t} g(u)\right) p$ 
subject to $-\left[\nabla\left(f(u)+y^{t} g(u)\right)+\nabla^{2}\left(f(u)+y^{t} g(u)\right) p\right] \in C_{1}^{*}$,

$u^{t}\left[\nabla\left(f(u)+y^{t} g(u)\right)+\nabla^{2}\left(f(u)+y^{t} g(u)\right) p\right] \leq 0$,

$y \in C_{2}$.

The purpose of this paper is to establish converse duality theorems between (P) and (ND1), (ND2), (ND3) and (ND4), respectively by invoking weak duality (Theorems 1-4 in [4]).

\section{Notations and preliminaries}

Let $R^{n}$ be the n-dimensional Euclidean space and $R_{+}^{n}$ be its nonnegative orthant. We now have the following definitions.

Definition 1. A set $C$ of $R^{n}$ is called a cone, if for each $x \in C$ and $\lambda \geq 0, \lambda x \in C$. Moreover, if $C$ is convex, then it is convex cone.

Definition 2. $C^{*}=\left\{z \in R^{n} \mid z^{t} x \leq 0\right.$, for all $\left.x \in C\right\}$ is called the polar of the cone $C$.

Let $C_{1} \subset R^{n}, C_{2} \subset R^{m}$ be closed convex cones with nonempty interiors having polars $C_{1}^{*}$ and $C_{2}^{*}$, respectively. Let $S \subseteq R^{n}$ be open and $C_{1} \subseteq S$ and let $f: S \rightarrow R$ and $g: S \rightarrow R^{m}$ be twice differentiable functions.

For other notations and preliminaries, we refer to Yang et al. [4].

\section{Second order converse duality}

In this section, we prove the following converse duality theorems for (ND1), (ND2), (ND3), and (ND4) respectively:

Theorem 1 (Converse duality). Let $(\bar{u}, \bar{y})$ be a local or global optimal solution of (ND1). Let

(i) the $n \times n$ Hessian matrix $\nabla^{2}\left(f(\bar{u})+\bar{y}^{t} g(\bar{u})\right)$ is nonsingular, and

(ii) the vector $\bar{p}^{t} \nabla\left(\nabla^{2} f(\bar{u}) \bar{p}+\nabla^{2} \bar{y}^{t} g(\bar{u}) \bar{p}\right)=0$ implies $\bar{p}=0$.

Then $\bar{u}$ is a feasible solution of (P) and the objective values of (P) and (ND1) are equal.

Also, if the hypotheses of weak duality (Theorem 1 in [4]) are satisfied for all feasible solutions of (P) and (ND1), then $\bar{u}$ is an optimal solution of (P).

Proof. Since $(\bar{u}, \bar{y})$ is a local or global optimal solution of (ND1), then by the Fritz John conditions [1], there exist $\alpha \in R_{+}$and $\beta \in C_{1}$ such that for each $y \in C_{2}$

$$
(\beta-\alpha \bar{u})^{t}\left[\nabla^{2} f(\bar{u})+\nabla^{2} \bar{y}^{t} g(\bar{u})+\nabla\left(\nabla^{2} f(\bar{u}) \bar{p}+\nabla^{2} \bar{y}^{t} g(\bar{u}) \bar{p}\right)\right]
$$




$$
\begin{aligned}
& -\frac{1}{2} \alpha \bar{p}^{t} \nabla\left(\nabla^{2} f(\bar{u}) \bar{p}+\nabla^{2} \bar{y}^{t} g(\bar{u}) \bar{p}\right)-\alpha\left[\nabla^{2} f(\bar{u}) \bar{p}+\nabla^{2} \bar{y}^{t} g(\bar{u}) \bar{p}\right]=0, \\
& {\left[\alpha\left(g(\bar{u})-\frac{1}{2} \bar{p}^{t} \nabla^{2} g(\bar{u}) \bar{p}\right)+(\beta-\alpha \bar{u})^{t}\left[\nabla g(\bar{u})+\nabla^{2} g(\bar{u}) \bar{p}\right]\right]^{t}(y-\bar{y}) \leq 0, \quad \forall y \in C_{2},} \\
& (\beta-\alpha \bar{u}-\alpha \bar{p})^{t}\left[\nabla^{2} f(\bar{u})+\nabla^{2} \bar{y}^{t} g(\bar{u})\right]=0,(3) \\
& \beta^{t}\left[\nabla f(\bar{u})+\nabla \bar{y}^{t} g(\bar{u})+\nabla^{2} f(\bar{u}) \bar{p}+\nabla^{2} \bar{y}^{t} g(\bar{u}) \bar{p}\right]=0,(4) \\
& (\alpha, \beta) \neq 0 .(5)
\end{aligned}
$$

Since $\nabla^{2}\left(f(\bar{u})+\bar{y}^{t} g(\bar{u})\right)$ is nonsingular, we have from (3)

$$
\beta=\alpha \bar{u}+\alpha \bar{p} .
$$

We claim that $\alpha \neq 0$. Indeed, if $\alpha=0$, then from (6), $\beta=0$. Thus $(\alpha, \beta)=0$, a contradiction to (5). Hence $\alpha>0$.

Using (6) in (1), we get

$$
\frac{1}{2} \alpha \bar{p}^{t} \nabla\left(\nabla^{2} f(\bar{u}) \bar{p}+\nabla^{2} \bar{y}^{t} g(\bar{u}) \bar{p}\right)=0,
$$

which along with assumption $(i i)$ and $\alpha>0$ implies $\bar{p}=0$. When $\bar{p}=0$, (6) reduces to

$$
\beta=\alpha \bar{u}
$$

Therefore, we have

$$
\bar{u}=\frac{\beta}{\alpha} \in C_{1} .
$$

Using (7) in (2) along with $\alpha>0$, and $\bar{p}=0$, we have

$$
[g(\bar{u})]^{t}(y-\bar{y}) \leq 0, \forall y \in C_{2} .
$$

Let $y \in C_{2}$. Then $y+\bar{y} \in C_{2}$, as $C_{2}$ is a closed convex cone, and so (9) shows that for every $y \in C_{2}, y^{t} g(\bar{u}) \leq 0$,

which implies

$$
g(\bar{u}) \in C_{2}^{*} .
$$

Thus, from (8) and (10), it follows that $\bar{u}$ is feasible for (P). Also, by letting $y=0$ and $y=2 \bar{y}$, simultaneously in (9), we get

$$
\bar{y}^{t} g(\bar{u})=0 \text {. }
$$

From (4), (7), (11), $\bar{p}=0$ and $\alpha>0$, it follows that

$$
\begin{gathered}
f(\bar{u})=f(\bar{u})+\bar{y}^{t} g(\bar{u})-\frac{1}{2} \bar{p}^{t} \nabla^{2}\left(f(\bar{u})+\bar{y}^{t} g(\bar{u})\right) \bar{p} \\
-\bar{u}^{t}\left[\nabla\left(f(\bar{u})+\bar{y}^{t} g(\bar{u})\right)+\nabla^{2}\left(f(\bar{u})+\bar{y}^{t} g(\bar{u})\right) \bar{p}\right] .
\end{gathered}
$$

That is, the objective values of (P) and (ND1) are equal. The optimality of $\bar{u}$ for (P) thus follows from weak duality (Theorem 1 in [4]). 
Theorem 2 (Converse duality). Let $(\bar{u}, \bar{y})$ be a local or global optimal solution of (ND2). Let

(i) either (a) $\nabla^{2} f(\bar{u})$ is positive definite and $\bar{p}^{t} \nabla f(\bar{u}) \geq 0$ or (b) $\nabla^{2} f(\bar{u})$ is negative definite and $\bar{p}^{t} \nabla f(\bar{u}) \leq 0$,

(ii) the vectors $\left\{\left[\nabla^{2} f(\bar{u})\right]_{j},\left[\nabla^{2} \bar{y}^{t} g(\bar{u})\right]_{j}, j=1,2, \ldots, n\right\}$ are linearly independent, where $\left[\nabla^{2} f(\bar{u})\right]_{j}$ is the $j^{\text {th }}$ row of $\nabla^{2} f(\bar{u})$ and $\left[\nabla^{2} \bar{y}^{t} g(\bar{u})\right]_{j}$ is the $j^{\text {th }}$ row of $\nabla^{2} \bar{y}^{t} g(\bar{u})$, and

(iii) $\nabla f(\bar{u}) \neq 0$.

Then $\bar{u}$ is a feasible solution of (P) and the objective values of (P) and (ND2) are equal.

Also, if the hypotheses of weak duality (Theorem 2 in [4]) are satisfied for all feasible solutions of $(\mathrm{P})$ and (ND2), then $\bar{u}$ is an optimal solution of $(\mathrm{P})$.

Proof. Since $(\bar{u}, \bar{y})$ is a local or global optimal solution of (ND2), then by the Fritz John conditions [1], there exist $\alpha \in R_{+}, \beta \in C_{1}$ and $\lambda \in R_{+}$such that for each $y \in C_{2}$

$$
\begin{aligned}
& (\beta-\lambda \bar{u})^{t}\left[\nabla^{2} f(\bar{u})+\nabla^{2} \bar{y}^{t} g(\bar{u})+\nabla\left(\nabla^{2} f(\bar{u}) \bar{p}+\nabla^{2} \bar{y}^{t} g(\bar{u}) \bar{p}\right)\right]-\frac{1}{2} \alpha \bar{p}^{t} \nabla\left(\nabla^{2} f(\bar{u}) \bar{p}\right) \\
& -\frac{1}{2} \lambda \bar{p}^{t} \nabla\left(\nabla^{2} \bar{y}^{t} g(\bar{u}) \bar{p}\right)-\lambda \bar{p}^{t}\left[\nabla^{2} f(\bar{u})+\nabla^{2} \bar{y}^{t} g(\bar{u})\right]+(\alpha-\lambda) \nabla f(\bar{u})=0, \quad(12) \\
& {\left[\lambda\left(g(\bar{u})-\frac{1}{2} \bar{p}^{t} \nabla^{2} g(\bar{u}) \bar{p}\right)+(\beta-\lambda \bar{u})^{t}\left[\nabla g(\bar{u})+\nabla^{2} g(\bar{u}) \bar{p}\right]\right]^{t}(y-\bar{y}) \leq 0, \forall y \in C_{2},} \\
& (\beta-\lambda \bar{u}-\alpha \bar{p})^{t} \nabla^{2} f(\bar{u})+(\beta-\lambda \bar{u}-\lambda \bar{p})^{t} \nabla^{2} \bar{y}^{t} g(\bar{u})=0, \quad(14) \\
& \beta^{t}\left[\nabla f(\bar{u})+\nabla \bar{y}^{t} g(\bar{u})+\nabla^{2} f(\bar{u}) \bar{p}+\nabla^{2} \bar{y}^{t} g(\bar{u}) \bar{p}\right]=0,(15) \\
& \lambda\left[\bar{y}^{t} g(\bar{u})-\frac{1}{2} \bar{p}^{t} \nabla^{2} \bar{y}^{t} g(\bar{u}) \bar{p}-\bar{u}^{t}\left[\nabla\left(f(\bar{u})+\bar{y}^{t} g(\bar{u})\right)+\nabla^{2}\left(f(\bar{u})+\bar{y}^{t} g(\bar{u})\right) \bar{p}\right]\right]=0, \\
& (\alpha, \beta, \lambda) \neq 0 .(17)
\end{aligned}
$$

Because of assumption (ii), (14) gives

$$
\begin{aligned}
& \beta=\lambda \bar{u}+\alpha \bar{p}, \\
& \beta=\lambda \bar{u}+\lambda \bar{p} .
\end{aligned}
$$

Using (18) and (19) in (12), we get

$$
\frac{1}{2}(\beta-\lambda \bar{u})^{t}\left[\nabla\left(\nabla^{2} f(\bar{u}) \bar{p}+\nabla^{2} \bar{y}^{t} g(\bar{u}) \bar{p}\right)\right]+(\alpha-\lambda) \nabla f(\bar{u})=0 .
$$

Let $\alpha=0$. Then, from (18), we have $\beta=\lambda \bar{u}$, and therefore (20) yields

$$
(\alpha-\lambda) \nabla f(\bar{u})=0,
$$

which on using assumption ( iii) gives $\lambda=\alpha=0$, and therefore, we have $\beta=0$. Thus, $(\alpha, \beta, \lambda)=0$, a contradiction to (17). Hence, $\alpha>0$. 
Letting $y=0$ and $y=2 \bar{y}$, simultaneously in (13), we get

$$
\lambda\left[\bar{y}^{t} g(\bar{u})-\frac{1}{2} \bar{p}^{t} \nabla^{2} \bar{y}^{t} g(\bar{u}) \bar{p}\right]+(\beta-\lambda \bar{u})^{t}\left[\nabla \bar{y}^{t} g(\bar{u})+\nabla^{2} \bar{y}^{t} g(\bar{u}) \bar{p}\right]=0 .
$$

From (15) and (16), it follows that

$$
(\beta-\lambda \bar{u})^{t}\left[\nabla f(\bar{u})+\nabla \bar{y}^{t} g(\bar{u})+\nabla^{2} f(\bar{u}) \bar{p}+\nabla^{2} \bar{y}^{t} g(\bar{u}) \bar{p}\right]+\lambda\left[\bar{y}^{t} g(\bar{u})-\frac{1}{2} \bar{p}^{t} \nabla^{2} \bar{y}^{t} g(\bar{u}) \bar{p}\right]=0 .
$$

Subtracting (21) from (22) to get

$$
(\beta-\lambda \bar{u})^{t}\left[\nabla f(\bar{u})+\nabla^{2} f(\bar{u}) \bar{p}\right]=0,
$$

which on using (18) along with $\alpha>0$ yields

$$
\bar{p}^{t}\left[\nabla f(\bar{u})+\nabla^{2} f(\bar{u}) \bar{p}\right]=0 .
$$

We claim that $\bar{p}=0$. Otherwise, either $(a)$ or $(b)$ of assumption $(i)$ implies

$$
\bar{p}^{t}\left[\nabla f(\bar{u})+\nabla^{2} f(\bar{u}) \bar{p}\right] \neq 0,
$$

contradicting (23). Hence $\bar{p}=0$. So (18) and (19) give

$$
\beta=\lambda \bar{u} \text {. }
$$

This together with (20), $\alpha>0$ and using hypothesis (iii) yields $\lambda=\alpha>0$. Therefore, it follows from (24) that

$$
\bar{u}=\frac{\beta}{\lambda} \in C_{1} .
$$

Using (24) in (13) with $\lambda>0$ and $\bar{p}=0$, we get

$$
[g(\bar{u})]^{t}(y-\bar{y}) \leq 0, \forall y \in C_{2} .
$$

Let $y \in C_{2}$. Then $y+\bar{y} \in C_{2}$, as $C_{2}$ is a closed convex cone and so (26) shows that for every $y \in C_{2}, y^{t} g(\bar{u}) \leq 0$, which implies

$$
g(\bar{u}) \in C_{2}^{*} \text {. }
$$

Thus, from (25) and (27), it follows that $\bar{u}$ is feasible for $(\mathrm{P})$. Since $\bar{p}=0$, we have

$$
f(\bar{u})=f(\bar{u})-\frac{1}{2} \bar{p}^{t} \nabla^{2} f(\bar{u}) \bar{p} .
$$

That is, the objective values of (P) and (ND2) are equal. Optimality of $\bar{u}$ for (P) thus follows from weak duality (Theorem 2 in [4]).

Theorem 3 (Converse duality). Let $(\bar{u}, \bar{y})$ be a local or global optimal solution of (ND3). Let

(i) either (a) $\nabla^{2} y^{t} g(\bar{u})$ is positive definite and $\bar{p}^{t} \nabla y^{t} g(\bar{u}) \geq 0$ or (b) $\nabla^{2} y^{t} g(\bar{u})$ is negative definite and $\bar{p}^{t} \nabla y^{t} g(\bar{u}) \leq 0$,

(ii) the vectors $\left\{\left[\nabla^{2} f(\bar{u})\right]_{j},\left[\nabla^{2} \bar{y}^{t} g(\bar{u})\right]_{j}, j=1,2, \ldots, n\right\}$ are linearly independent, where $\left[\nabla^{2} f(\bar{u})\right]_{j}$ is the $j^{\text {th }}$ row of $\nabla^{2} f(\bar{u})$ and $\left[\nabla^{2} \bar{y}^{t} g(\bar{u})\right]_{j}$ is the $j^{\text {th }}$ row of $\nabla^{2} \bar{y}^{t} g(\bar{u})$, and

(iii) $\nabla y^{t} g(\bar{u}) \neq 0$. 
Then $\bar{u}$ is a feasible solution of $(\mathrm{P})$ and the objective values of $(\mathrm{P})$ and (ND3) are equal.

Also, if the hypotheses of weak duality (Theorem 3 in [4]) are satisfied for all feasible solutions of (P) and (ND3), then $\bar{u}$ is an optimal solution of (P).

Proof. Since $(\bar{u}, \bar{y})$ is a local or global optimal solution of (ND3), then by the Fritz John conditions [1], there exist $\alpha \in R_{+}, \beta \in C_{1}$ and $\lambda \in R_{+}$such that for each $y \in C_{2}$

$$
\begin{aligned}
& (\beta-\alpha \bar{u})^{t}\left[\nabla^{2} f(\bar{u})+\nabla^{2} \bar{y}^{t} g(\bar{u})+\nabla\left(\nabla^{2} f(\bar{u}) \bar{p}+\nabla^{2} \bar{y}^{t} g(\bar{u}) \bar{p}\right)\right]-\frac{1}{2} \alpha \bar{p}^{t} \nabla\left(\nabla^{2} f(\bar{u}) \bar{p}\right) \\
& -\frac{1}{2} \lambda \bar{p}^{t} \nabla\left(\nabla^{2} \bar{y}^{t} g(\bar{u}) \bar{p}\right)-\alpha \bar{p}^{t}\left[\nabla^{2} f(\bar{u})+\nabla^{2} \bar{y}^{t} g(\bar{u})\right]+(\lambda-\alpha) \nabla y^{t} g(\bar{u})=0, \quad(28) \\
& {\left[\lambda\left(g(\bar{u})-\frac{1}{2} \bar{p}^{t} \nabla^{2} g(\bar{u}) \bar{p}\right)+(\beta-\alpha \bar{u})^{t}\left[\nabla g(\bar{u})+\nabla^{2} g(\bar{u}) \bar{p}\right]\right]^{t}(y-\bar{y}) \leq 0, \forall y \in C_{2}, \quad(29)} \\
& (\beta-\alpha \bar{u}-\alpha \bar{p})^{t} \nabla^{2} f(\bar{u})+(\beta-\alpha \bar{u}-\lambda \bar{p})^{t} \nabla^{2} \bar{y}^{t} g(\bar{u})=0, \quad(30) \\
& \beta^{t}\left[\nabla f(\bar{u})+\nabla \bar{y}^{t} g(\bar{u})+\nabla^{2} f(\bar{u}) \bar{p}+\nabla^{2} \bar{y}^{t} g(\bar{u}) \bar{p}\right]=0, \quad(31) \\
& \lambda\left[\bar{y}^{t} g(\bar{u})-\frac{1}{2} \bar{p}^{t} \nabla^{2} \bar{y}^{t} g(\bar{u}) \bar{p}\right]=0,(32) \\
& (\alpha, \beta, \lambda) \neq 0 .(33)
\end{aligned}
$$

Because of assumption (ii), (30) gives

$$
\begin{aligned}
& \beta=\alpha \bar{u}+\alpha \bar{p}, \\
& \beta=\alpha \bar{u}+\lambda \bar{p} .
\end{aligned}
$$

Using (34) and (35) in (28), we get

$$
\frac{1}{2}(\beta-\alpha \bar{u})^{t}\left[\nabla\left(\nabla^{2} f(\bar{u}) \bar{p}+\nabla^{2} \bar{y}^{t} g(\bar{u}) \bar{p}\right)\right]+(\lambda-\alpha) \nabla f(\bar{u})=0 .
$$

Let $\lambda=0$. Then, from (35), we have $\beta=\alpha \bar{u}$, and therefore (36) yields

$$
(\lambda-\alpha) \nabla \bar{y}^{t} g(\bar{u})=0,
$$

which on using assumption (iii) gives $\alpha=\lambda=0$, and therefore, we have $\beta=0$. Thus, $(\alpha, \beta, \lambda)=0$, a contradiction to (33). Hence, $\lambda>0$.

Letting $y=0$ and $y=2 \bar{y}$, simultaneously in (29), we get

$$
\lambda\left[\bar{y}^{t} g(\bar{u})-\frac{1}{2} \bar{p}^{t} \nabla^{2} \bar{y}^{t} g(\bar{u}) \bar{p}\right]+(\beta-\alpha \bar{u})^{t}\left[\nabla \bar{y}^{t} g(\bar{u})+\nabla^{2} \bar{y}^{t} g(\bar{u}) \bar{p}\right]=0 .
$$

Subtracting (32) from (37) to get

$$
(\beta-\alpha \bar{u})^{t}\left[\nabla \bar{y}^{t} g(\bar{u})+\nabla^{2} \bar{y}^{t} g(\bar{u}) \bar{p}\right]=0,
$$

which on using (35) along with $\lambda>0$ yields

$$
\bar{p}^{t}\left[\nabla \bar{y}^{t} g(\bar{u})+\nabla^{2} \bar{y}^{t} g(\bar{u}) \bar{p}\right]=0 .
$$


We claim that $\bar{p}=0$. Otherwise, either $(a)$ or $(b)$ of assumption $(i)$ implies

$$
\bar{p}^{t}\left[\nabla \bar{y}^{t} g(\bar{u})+\nabla^{2} \bar{y}^{t} g(\bar{u}) \bar{p}\right] \neq 0,
$$

contradicting (38). Hence $\bar{p}=0$. So (34) and (35) give

$$
\beta=\alpha \bar{u} \text {. }
$$

This together with (36), $\lambda>0$ and using hypothesis (iii) yields $\alpha=\lambda>0$. Therefore, it follows from (39) that

$$
\bar{u}=\frac{\beta}{\alpha} \in C_{1} .
$$

Using (39) in (29) with $\lambda>0$ and $\bar{p}=0$, we get

$$
[g(\bar{u})]^{t}(y-\bar{y}) \leq 0, \forall y \in C_{2} .
$$

Let $y \in C_{2}$. Then $y+\bar{y} \in C_{2}$, as $C_{2}$ is a closed convex cone and so (41) shows that for every $y \in C_{2}, y^{t} g(\bar{u}) \leq 0$, which implies

$$
g(\bar{u}) \in C_{2}^{*} .
$$

Thus, from (40) and (42), it follows that $\bar{u}$ is feasible for (P). Also, from (31), (39), $\alpha>0$ and $\bar{p}=0$, we have

$$
f(\bar{u})=f(\bar{u})-\frac{1}{2} \bar{p}^{t} \nabla^{2} f(\bar{u}) \bar{p}-\bar{u}^{t}\left[\nabla f(\bar{u})+\nabla \bar{y}^{t} g(\bar{u})+\nabla^{2} f(\bar{u}) \bar{p}+\nabla^{2} \bar{y}^{t} g(\bar{u}) \bar{p}\right] .
$$

That is, the objective values of (P) and (ND3) are equal. Optimality of $\bar{u}$ for (P) thus follows from weak duality (Theorem 3 in [4]).

Theorem 4 (Converse duality). Let $(\bar{u}, \bar{y})$ be a local or global optimal solution of (ND4). Let

(i) either (a) $\nabla^{2}\left(f(\bar{u})+\bar{y}^{t} g(\bar{u})\right)$ is positive definite and $\bar{p}^{t} \nabla\left(f(\bar{u})+\bar{y}^{t} g(\bar{u})\right) \geq 0$ or (b) $\nabla^{2}\left(f(\bar{u})+\bar{y}^{t} g(\bar{u})\right)$ is negative definite and $\bar{p}^{t} \nabla\left(f(\bar{u})+\bar{y}^{t} g(\bar{u})\right) \leq 0$, and

(ii) $\nabla f(\bar{u})+\nabla \bar{y}^{t} g(\bar{u})+\nabla^{2} f(\bar{u}) \bar{p}+\nabla^{2} \bar{y}^{t} g(\bar{u}) \bar{p} \neq 0$.

Then $\bar{u}$ is a feasible solution of (P) and the objective values of (P) and (ND4) are equal.

Also, if the hypotheses of weak duality (Theorem 4 in [4]) are satisfied for all feasible solutions of (P) and (ND4), then $\bar{u}$ is an optimal solution of (P).

Proof. Since $(\bar{u}, \bar{y})$ is a local or global optimal solution of (ND2), then by the Fritz John conditions [1], there exist $\alpha \in R_{+}, \beta \in C_{1}$ and $\lambda \in R_{+}$such that for each $y \in C_{2}$

$$
\begin{aligned}
& (\beta-\lambda \bar{u})^{t}\left[\nabla^{2} f(\bar{u})+\nabla^{2} \bar{y}^{t} g(\bar{u})+\nabla\left(\nabla^{2} f(\bar{u}) \bar{p}+\nabla^{2} \bar{y}^{t} g(\bar{u}) \bar{p}\right)\right]-\frac{1}{2} \alpha \bar{p}^{t} \nabla\left(\nabla^{2} f(\bar{u}) \bar{p}+\right. \\
& \left.\nabla^{2} \bar{y}^{t} g(\bar{u}) \bar{p}\right) \\
& -\lambda \bar{p}^{t}\left[\nabla^{2} f(\bar{u})+\nabla^{2} \bar{y}^{t} g(\bar{u})\right]+(\alpha-\lambda)\left[\nabla f(\bar{u})+\nabla \bar{y}^{t} g(\bar{u})\right]=0,
\end{aligned}
$$




$$
\begin{aligned}
& {\left[\alpha\left(g(\bar{u})-\frac{1}{2} \bar{p}^{t} \nabla^{2} g(\bar{u}) \bar{p}\right)+(\beta-\lambda \bar{u})^{t}\left[\nabla g(\bar{u})+\nabla^{2} g(\bar{u}) \bar{p}\right]\right]^{t}(y-\bar{y}) \leq 0, \forall y \in C_{2},} \\
& (\beta-\lambda \bar{u}-\alpha \bar{p})^{t}\left[\nabla^{2} f(\bar{u})+\nabla^{2} \bar{y}^{t} g(\bar{u})\right]=0,(45) \\
& \beta^{t}\left[\nabla f(\bar{u})+\nabla \bar{y}^{t} g(\bar{u})+\nabla^{2} f(\bar{u}) \bar{p}+\nabla^{2} \bar{y}^{t} g(\bar{u}) \bar{p}\right]=0, \quad(46) \\
& \lambda \bar{u}^{t}\left[\nabla f(\bar{u})+\nabla \bar{y}^{t} g(\bar{u})+\nabla^{2} f(\bar{u}) \bar{p}+\nabla^{2} \bar{y}^{t} g(\bar{u}) \bar{p}\right]=0,(47) \\
& (\alpha, \beta, \lambda) \neq 0 .(48)
\end{aligned}
$$

By assumption $(i), \nabla^{2}\left(f(\bar{u})+\bar{y}^{t} g(\bar{u})\right)$ is obviously nonsingular, and so (45) gives

$$
\beta=\lambda \bar{u}+\alpha \bar{p},
$$

We laim that $\alpha \neq 0$. Indeed, if $\alpha=0$, we have

$$
\beta=\lambda \bar{u}
$$

which along with (43) implies

$$
\lambda\left[\nabla f(\bar{u})+\nabla \bar{y}^{t} g(\bar{u})+\nabla^{2} f(\bar{u}) \bar{p}+\nabla^{2} \bar{y}^{t} g(\bar{u}) \bar{p}\right]=0 .
$$

On using assumption (ii), we have $\lambda=0$, and therefore $\beta=0$. Thus, $(\alpha, \beta, \lambda)=0$, a contradiction to (48). Hence, $\alpha>0$.

From (46) and (47), we have

$$
(\beta-\lambda \bar{u})^{t}\left[\nabla f(\bar{u})+\nabla \bar{y}^{t} g(\bar{u})+\nabla^{2} f(\bar{u}) \bar{p}+\nabla^{2} \bar{y}^{t} g(\bar{u}) \bar{p}\right]=0,
$$

which on using (49) along with $\alpha>0$ yields

$$
p^{t}\left[\nabla f(\bar{u})+\nabla \bar{y}^{t} g(\bar{u})+\nabla^{2} f(\bar{u}) \bar{p}+\nabla^{2} \bar{y}^{t} g(\bar{u}) \bar{p}\right]=0 .
$$

We claim that $\bar{p}=0$. Otherwise, either (a) or (b) of assumption $(i)$ implies

$$
p^{t}\left[\nabla f(\bar{u})+\nabla \bar{y}^{t} g(\bar{u})+\nabla^{2} f(\bar{u}) \bar{p}+\nabla^{2} \bar{y}^{t} g(\bar{u}) \bar{p}\right] \neq 0,
$$

contradicting (50). Hence $\bar{p}=0$. So (49) gives

$$
\beta=\lambda \bar{u}
$$

Equation (43) together with (51) and $\bar{p}=0$ implies

$$
(\alpha-\lambda)\left[\nabla f(\bar{u})+\nabla \bar{y}^{t} g(\bar{u})+\nabla^{2} f(\bar{u}) \bar{p}+\nabla^{2} \bar{y}^{t} g(\bar{u}) \bar{p}\right]=0,
$$

which on using assumption (ii) implies $\lambda=\alpha>0$. Therefore, it follows from (51) that

$$
\bar{u}=\frac{\beta}{\lambda} \in C_{1} .
$$

Using (51) in (44) with $\alpha>0$ and $\bar{p}=0$, we have

$$
[g(\bar{u})]^{t}(y-\bar{y}) \leq 0, \forall y \in C_{2} .
$$

Let $y \in C_{2}$. Then $y+\bar{y} \in C_{2}$, as $C_{2}$ is a closed convex cone and so (53) shows that for every $y \in C_{2}, y^{t} g(\bar{u}) \leq 0$, which implies

$$
g(\bar{u}) \in C_{2}^{*} .
$$


Thus, from (52) and (54), it follows that $\bar{u}$ is feasible for (P). Also, by letting $y=0$ and $y=2 \bar{y}$, simultaneously in (53), we get

$$
\bar{y}^{t} g(\bar{u})=0 \text {. }
$$

From (55) and $\bar{p}=0$, it follows

$$
f(\bar{u})=f(\bar{u})+\bar{y}^{t} g(\bar{u})-\frac{1}{2} \bar{p}^{t}\left(\nabla^{2} f(\bar{u})+\nabla^{2} \bar{y}^{t} g(\bar{u})\right) \bar{p} .
$$

That is, the objective values of (P) and (ND4) are equal. Optimality of $\bar{u}$ for (P) thus follows from weak duality (Theorem 4 in [4]).

\section{Conclusion}

From the applications point of view of second order duality that it provides tighter bounds than first order duality, the second order weak and strong duality results for more general problems with cone constraints discussed in [4] are very useful, but the converse duality theorems, which are the important part of duality relationships between primal and dual problems, are missing there. In this paper, we are intended to fill this gap by establishing converse duality theorems for four types of second order duals formulated in [4], however, we invoke the weak duality theorems proved therein.

\section{REFERENCES}

[1] M.S. Bazaraa, J.J. Goode, On symmetric duality in nonlinear programming, Oper. Res., 21 (1973), 1-9.

[2] S. Chandra, Abha, A note on pseudo-invexity and duality in nonlinear programming, European J. Oper. Res., 122 (2000), 161-165.

[3] L.N. Das, S. Nanda, Pseudo-invexity and duality in nonlinear programming, European J. Oper. Res., 88 (1996), 572-577.

[4] X.M. Yang, X.Q. Yang, K.L. Teo, S.H. Hou, Second order duality for nonlinear programming, Indian J. Pure Appl. Math., 35 (2004), 699-708.

[5] X.M. Yang, X.Q. Yang, K.L. Teo, Converse duality in nonlinear programming with cone constraints, European J. Oper. Res., 170 (2006), 350-354.

${ }^{1}$ Department of Mathematics and Statistics, King Fahd University of Petroleum \& Minerals, Dhahran-31261, Saudi Arabia

E-mail address: drizhar@kfupm.edu.sa (I. Ahmad)

E-mail address: agarwal@fit.edu (Ravi P. Agarwal) 